An official journal of the / Un journal officiel de la "Société Sénégalaise de Cancérologie" (SOSECAN)

Journal homepage: www.africanjournalofoncology.com

\title{
Cancers in Africa: a journal to participate in the fight
}

\section{Les cancers en Afrique : un journal pour participer à la lutte}

\author{
S. Ka ${ }^{\prime *}$, MM. Dieng', A. Dem'. \\ I Institut Joliot Curie de Dakar, Sénégal.
}

Le cancer n'épargne aucun continent, aucun âge, aucun sexe. Dans les projections de l'OMS, la transition épidémiologique fera de l'Afrique le continent le plus touché dans les décennies à venir [I]. Le fardeau du cancer est supporté par toutes les couches de la population africaine. Chez les enfants, on retrouve une grande fréquence des hémopathies malignes et des «blastomes ». La répartition de ces cancers est peu connue du fait de la rareté des données portant sur les cancers de l'enfant. Chez les femmes, les déterminants de l'éradication du cancer du col sont très peu maitrisés. L'épidémiologie est marquée par la courbe sans cesse croissante de l'incidence du cancer du sein qui de plus en plus occupe la première place dans les séries africaines. Le cancer de la prostate se heurte à un difficile accès aux moyens de prise en charge [2]. La mise en place de registres des cancers est timide dans les pays en développement notamment en Afrique. Ce qui pose le problème de la fiabilité des données régulièrement publiées. Dans le même contexte des situations de crise notamment sécuritaires, la malnutrition et les épidémies comme le $\mathrm{VIH}$ rendent plus difficile la problématique de la prise en charge des cancers [3]. La récente pandémie de la Covid-19 a porté un coup de grâce à des systèmes sanitaires à ressources limitées [4].

II y a une corrélation entre l'incidence des cancers et les revenus des populations. Les cancers liés au bas niveau socioéconomique sont fréquents en Afrique. La lutte contre le cancer se heurte aux impératifs sécuritaires et de la lutte pour l'émergence des économies africaines [5].

C'est dans ce contexte que les universités africaines évoluent avec l'impérieuse nécessité de relever leurs capacités d'enseignement, de recherche et d'innovation. II existe très peu de formations diplômantes dans les domaines clés de la cancérologie. En Afrique francophone au sud du Sahara, moins de deux centres enseignent l'oncologie médicale, la chirurgie oncologique et la radiothérapie. D'où la nécessité de reconsidérer les paradigmes de l'enseignement des spécialités liées au cancer dans les pays en développement [6].

Pendant que la prise en charge des cancers dans le monde bénéficie de l'essor de la prévention avec notamment la vaccination contre le HPV, de la découverte de nouveaux traitements telles que les thérapies ciblées, la prise en charge des cancers se fait quotidiennement en Afrique généralement au gré de la disponibilité de lits, de praticiens diplômés, d'équipements dédiés et de moyens médicaux disponibles et accessibles [5, 7]. Cependant, on assiste de plus en plus à l'érection de centres de cancérologie multidisciplinaire équipés de machines de radiothérapie. D'où la nécessaire adéquation entre les ressources humaines, les infrastructures et les équipements.

Devant les besoins en soins et la faiblesse des ressources, la recherche clinique et fondamentale a été reléguée au second plan. Son corollaire, la publication des résultats scientifiques, vivier des connaissances, outil de vulgarisation des activités scientifiques et de la des carrières des enseignants et des chercheurs, est devenue marginale.

L'accès à des journaux de spécialités en cancérologie est rendu difficile par leur rareté dans le monde et leurs critères d'accès difficilement concordant avec les réalités de la recherche en Afrique. Les journaux de spécialités s'adossent sur des sociétés savantes vivaces et dont le vivier est prolixe laissant peu de place aux concurrents d'autres contrées. Pourtant le cancer a été étudié et publié de tout temps par tous les précurseurs de la médecine moderne, de l'Egypte ancienne à travers le papyrus de Edwin à nos jours, en passant par De Medicina de Celcus en 1478, premier livre publié connu [8]. Il est donc temps de mettre en place des journaux et cadres, consensuels parce que multi- et transdisciplinaires, bi- ou multilingues couvrant les espaces scientifiques et politiques et surtout perspectivistes de par leurs ouvertures éditoriales et leurs évolutions.

Dans ce contexte est né l'African Journal of Oncology (AJO) ou le Journal Africain d'Oncologie (JAO).

\section{CONFLITS D'INTERET}

Les auteurs n'ont déclaré aucun conflit d'intérêts.

\section{REFERENCES}

I. Jemal A, Bray F, Center MM, Ferlay J, Ward E, Forman D. Global cancer statistics. CA Cancer J Clin 20I I;6I (2):69-90.

2. Jemal A, Bray F, Forman D, O'Brien M, Ferlay J, Center M, Parkin DM. Cancer burden in Africa and opportunities for prevention. Cancer 20I 2; I I 8(I8):4372-84.

3. Oh JK, Weiderpass E. Infection and cancer: global distribution and burden of diseases. Ann Glob Health 2014;80(5):384-92.

4. Nnaji CA, Moodley J. Impact of the COVID-19 pandemic on cancer diagnosis, treatment and research in African health systems: a review of 
current evidence and contextual perspectives. Ecancermedicalscience 202 I; 15: I 170.

5. Horton S, Gauvreau CL. Cancer in Low- and MiddleIncome Countries: An Economic Overview. In: Gelband H, Jha P, Sankaranarayanan R, Horton S, editors. Cancer: Disease Control Priorities, Third Edition (Volume 3). Washington (DC): The International Bank for Reconstruction and Development / The World Bank; 2015 Nov I. Chapter 16.

6. Amgad M, Shash E, Gaafar R. Cancer education for medical students in developing countries: where do we stand and how to improve? Crit Rev Oncol Hematol 2012;84(I): I22-9.

7. Busolo DS, Woodgate RL. Cancer prevention in Africa: a review of the literature. Glob Health Promot 20I5;22(2):3I-9.

8. Hajdu SI. A note from history: landmarks in history of cancer, part I. Cancer. 20 I I; I I7(5): I097-102. 